\title{
Living with Diabetes: The Right Approach ${ }^{1}$
}

\section{Nancy J. Gal and Linda B. Bobroff ${ }^{2}$}

\section{Introduction}

Diabetes is a disease that affects more than twenty million Americans. Although there is no cure, diabetes can be managed.

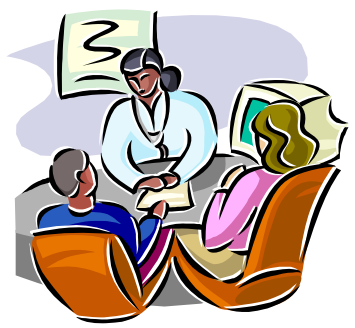

If you have diabetes, the best way to "live well" and prevent health problems is to learn as much as you can about the disease. You need to become an active part of your health care team, which includes you, a doctor, a nurse (LPN or RN), a registered dietitian (RD), a pharmacist, and a certified diabetes educator (CDE).

Your knowledge will help you to practice "healthy living" that includes:

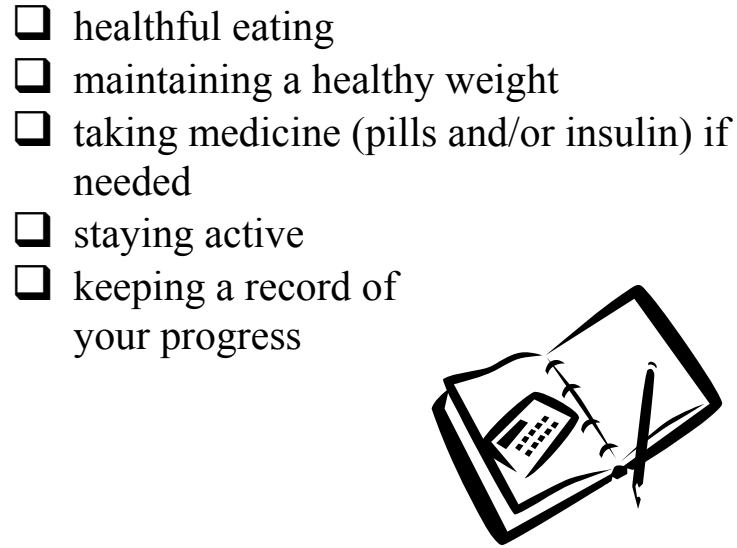

\section{What is diabetes?}

Diabetes is a disease in which your body is not able to use food properly. Diabetes causes glucose (sugar) to build up in your blood, which can lead to serious health problems. The general warning signs for diabetes are described in Table 1.

Table 1

\begin{tabular}{|l|}
\hline \multicolumn{1}{c|}{ General Warning Signs* } \\
$\square$ frequent urination \\
$\square$ increased thirst \\
$\square$ extreme hunger \\
$\square$ unusual and sudden weight loss \\
$\square$ dizziness \\
$\square$ blurred vision \\
$\square$ weakness and fatigue \\
$\square$ yeast infections \\
If you have any of these symptoms, see your \\
doctor right away to find out if you have \\
diabetes. If you do have diabetes, you can \\
reduce your risk of health complications by \\
starting treatment right away. \\
\hline *Onset of type 2 diabetes may have no \\
symptoms.
\end{tabular}

\footnotetext{
This document is FCS 8542, one in a series of the Department of Family, Youth and Community Sciences, Cooperative Extension Service, Institute of Food and Agricultural Sciences, University of Florida. Publication date: January 1996. Revised: October 2006. Please visit the EDIS Web site at http://edis.ifas.ufl.edu

Nancy J. Gal, MAg, Extension Agent IV, Marion County, Cooperative Extension Service, Linda B. Bobroff, PhD, RD, LD/N, professor, Department of Family, Youth and Community Sciences, Institute of Food and Agricultural Sciences, University of Florida. Reviewed by: Joy Semelka, MS, RD, LD/N, CDE; Sharon Valley, MS, CDE, University of Florida/Shands Diabetes Center; and Gail Kauwell, PhD, RD, LD/N, University of Florida.
}

2 
Normally, most of the food you eat is changed into glucose, which is the main energy source for the body. In order for your body to use this energy, the glucose must get into the cells.

Insulin, a hormone made in the pancreas, is the key that helps glucose get into your cells.

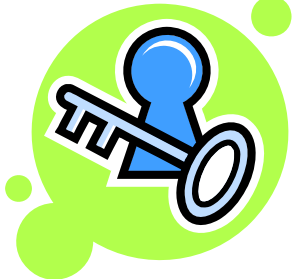

With diabetes, your body changes food into glucose, but the glucose either can't get into the cells or has difficulty getting into the cells. This is caused by insulin deficiency, insulin resistance, or both.

With insulin deficiency, the body cannot make insulin. People with type 1 diabetes are insulin deficient. Insulin resistance means that the body makes insulin, but not enough, or that the insulin that it does make does not work properly. This is type 2 diabetes. Some people with type 2 diabetes may also be insulin deficient. Both insulin deficiency and insulin resistance result in high blood glucose, since the glucose cannot get into the cells.

The long-term effects of high blood glucose levels include damage to blood vessels and nerves. This damage can cause very serious health problems. See Table 2 for a list of health problems that can occur when diabetes is not well controlled.

\section{Table 2}

\section{Possible Health Problems Related to Diabetes}

Eye disease (retinopathy)

Kidney disease (nephropathy)

Heart disease

Stroke

Pain or loss of feeling in legs and feet

(neuropathy)

Poor healing

Foot infections (sores)

\section{Who is at risk for diabetes?}

Untreated diabetes can lead to serious health problems. That is why it's important to have your blood glucose tested regularly. People who are at high risk need to be sure to be tested for diabetes. The following people are at high risk for getting type 2 diabetes:

- blood relatives of people with diabetes

- women who have had babies weighing nine pounds or more at birth

- women who have had gestational diabetes

- African Americans, Hispanics, and Native Americans

- people with impaired fasting glucose (see next section)

- people with high blood pressure

- people with very high blood cholesterol or triglyceride levels

- people who are obese

- persons over age 45 (risk increases with age)

- people who do not exercise regularly

\section{Understanding your blood glucose values}

The purpose of a blood glucose test is to learn the amount of glucose in your blood. There are two types of blood glucose tests: screening and diagnostic tests. They are not the same and have different purposes.

Screening tests are quick and inexpensive. They are performed on people who have no symptoms of diabetes. The test is done with a small drop of blood from the fingertip. Results are read from a meter in seconds. These tests often are done at health fairs.

Diagnostic blood tests are lab tests that use blood from a vein. They are generally done to confirm a diagnosis based on a person's symptoms. The diagnostic tests used to diagnose diabetes and pre-diabetes are the fasting plasma glucose test and oral glucose tolerance tests. 
The most common blood test for diagnosing diabetes is the fasting plasma glucose test. It is convenient, easy, and very reliable. The test is done at your doctor's office or a laboratory. It uses a sample of blood taken from a vein after an overnight fast (at least eight hours).

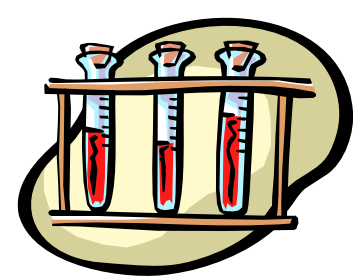

Normal fasting plasma glucose levels are 70 to 99 milligrams per deciliter of blood (mg/dl). Fasting plasma glucose levels equal to or more than $126 \mathrm{mg} / \mathrm{dl}$ from two or more tests indicate diabetes. Levels of 100 to $125 \mathrm{mg} / \mathrm{dl}$ indicate impaired fasting glucose or "pre-diabetes."

\begin{tabular}{|ll|}
\hline \multicolumn{2}{|c|}{ Fasting Plasma Glucose } \\
Normal: & $70-99 \mathrm{mg} / \mathrm{dl}$ \\
Pre-diabetes: & $100-125 \mathrm{mg} / \mathrm{dl}$ \\
Diabetes: & $126 \mathrm{mg} / \mathrm{dl}$ or higher \\
\hline
\end{tabular}

In diagnosing diabetes, your doctor considers not only your blood glucose values, but also your symptoms, overall health, and medical history.

Another blood test that may be used to diagnose diabetes (but not pre-diabetes) is the casual plasma glucose test. This blood test is done at random. It does not matter when the person last ate or drank. A casual plasma glucose test should only be done if classic diabetes symptoms are present. These include excessive urine, excessive thirst, and unexplained weight loss. A diagnosis of diabetes with this test requires evidence of these symptoms of diabetes.

\section{Types of diabetes}

There are four main types of diabetes.

- Type 1

- Gestational
- Type 2

- Secondary

\section{Type 1 diabetes}

This type occurs when the pancreas stops making insulin or when it makes a very small amount. Type 1 diabetes usually develops before the age of thirty, but can happen at any age.

Approximately one in ten people with diabetes has type 1 . People with type 1 diabetes must take insulin to live. Healthful eating and exercising also are important to their care.

\section{Type 2 diabetes}

This type of diabetes usually occurs when the pancreas doesn't make enough insulin or the insulin it makes doesn't work properly. This is called insulin resistance.

Type 2 diabetes is most common in people over the age of fortyfive, but also can happen at any age. It often runs in families.

Type 2 diabetes is more common among some ethnic groups,

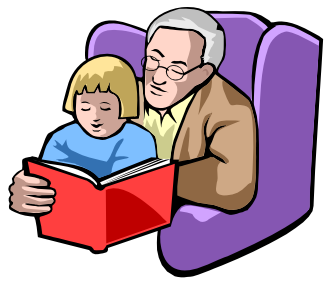
including African Americans, Hispanics, and Native Americans. Approximately nine out of ten people with diabetes have type 2 .

People with type 2 diabetes may be able to manage their disease by eating a healthful diet, achieving and maintaining a healthy weight, and exercising. Many persons with type 2 diabetes also must take pills and/or insulin.

\section{Gestational diabetes}

This type of diabetes occurs during pregnancy and usually goes away after the baby is born. However, women who have had gestational diabetes have a greater chance of getting type 2 diabetes later in life. They need to maintain a healthy lifestyle to reduce their risk of diabetes.

\section{Secondary diabetes}

This type of diabetes is caused by certain diseases, medical conditions, or medications 
that affect how insulin is made and used in the body. Certain drugs (steroids) commonly used for asthma and allergies, as well as organ transplants, can cause diabetes to occur. The diabetes may improve or go away once the treatment is complete or when less medicine is needed.

\section{Living with diabetes}

Living with diabetes is simply healthy living. Since there is no cure yet, the goal is to keep your blood glucose levels as close to normal as safe and appropriate. This will help you feel better today, and help reduce the risk of serious health problems in the future.

We now know that keeping blood glucose levels in good control (as close to normal as appropriate for the individual) helps prevent or delay complications of diabetes and related health problems. To keep your blood glucose levels as close to the normal range as possible, you need to take good care of yourself by:

- eating a healthful diet

- maintaining a healthy weight

- taking medicine if needed

- recording blood glucose levels

- staying active

- staying well informed and motivated

Let's see how you can do each of these to live well with diabetes.

\section{Healthful Eating}

Healthful eating is one way you can help control your blood glucose levels. Remember, the closer to normal your blood glucose levels stay, the lower your chances of having major health problems later. Eating a healthful diet will help you:

- control blood glucose levels

- manage your weight

- feel good

- improve your general health
There is no one diabetic diet. You simply follow a personal plan of healthful eating that is based on your own needs. Your plan will help you make healthy food choices, in appropriate amounts, and at the right time.

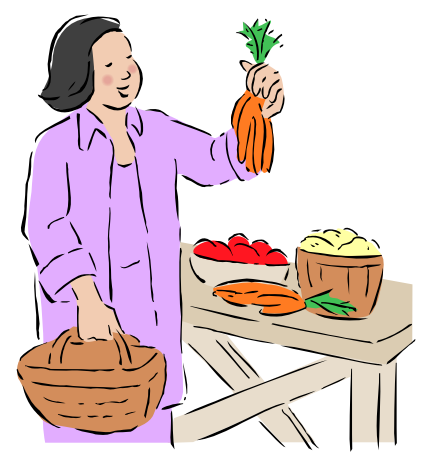

Your eating plan is based on:

- your blood glucose readings

- your weight

- the type and amount of medicine you take

- your activity level

- your general health

- the foods and beverages you enjoy

Healthful eating means eating a variety of healthy foods that you enjoy in appropriate amounts. Making healthy food choices from the Diabetes Food Pyramid food groups is a good start (see Figure 1). You and your family can follow the same guidelines, although you will need to carefully manage how much and when you eat.

Since your meal plan is so very important, it should be planned carefully. A registered dietitian and certified diabetes educator can help write a meal plan for you that includes:

- appropriate portion sizes

- timing of meals

- healthful food choices

- basic and advanced carbohydrate counting 


\section{Diabetes Food Pyramid}

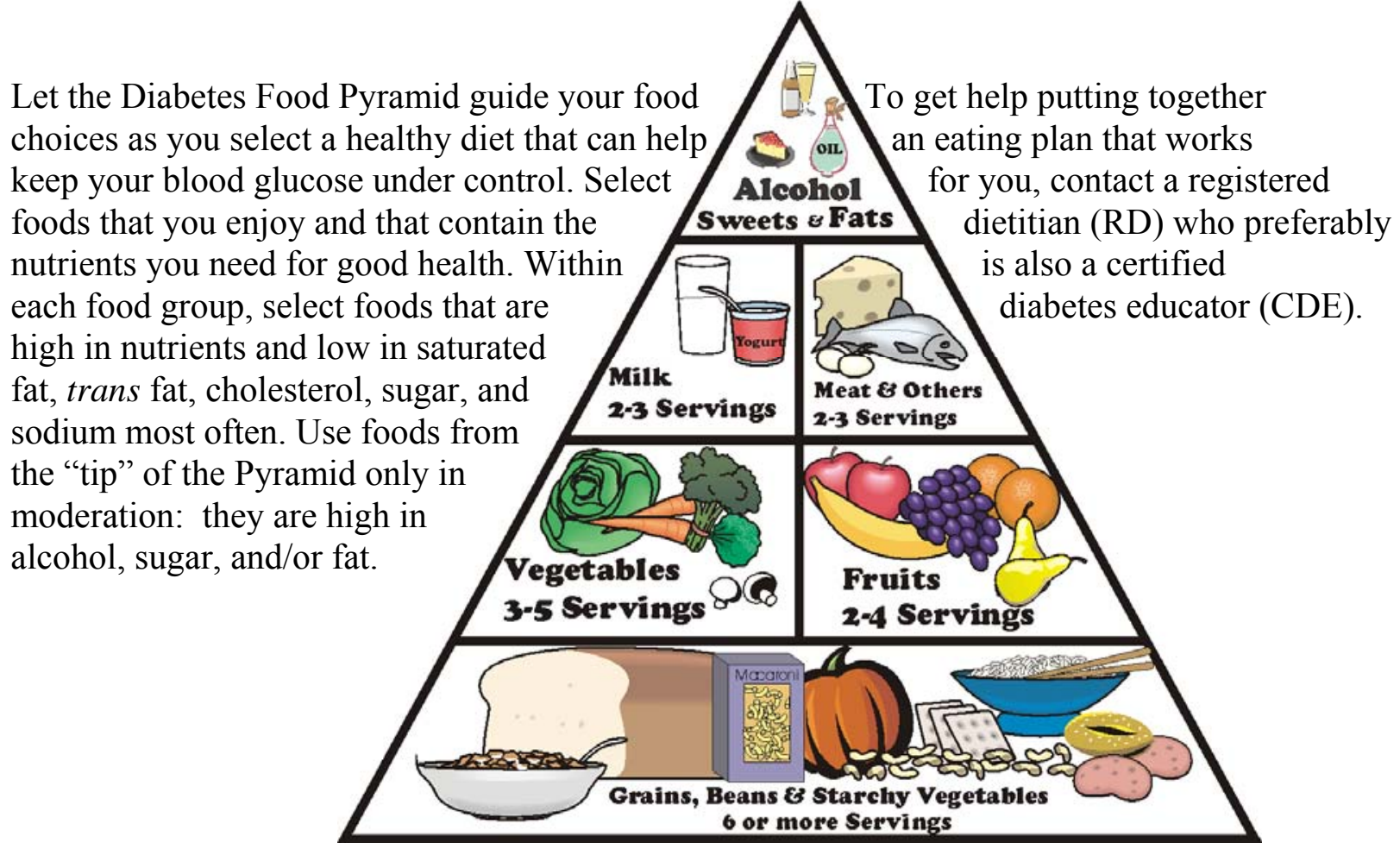

Figure 1

This Diabetes Food Pyramid is based on the USDA's original Food Guide Pyramid. It includes recommendations of the American Diabetes Association (ADA). Because foods in this pyramid are grouped based on their carbohydrate content, starchy vegetables and legumes are included in the Grains group, and cheese is included in the Meat group. To date, ADA has not changed their pyramid to reflect USDA's MyPyramid food guidance system (see http://MyPyramid.gov for information on this new food guide).

Reading food labels will help you select foods that fit your meal plan. The Nutrition Facts panel, found on almost all foods, will give you information on the carbohydrate, fat, and protein content of foods, as well as the calorie level per serving. You also can learn about other nutrients that are provided by the food.

Each nutrient value is given "per serving," and the serving size for that food is listed on the Nutrition Facts panel at the top. Always check the serving size, since the nutrients YOU will be getting depend on how much of the food you eat. For people with diabetes, the most important nutrient to keep track of is total carbohydrate.

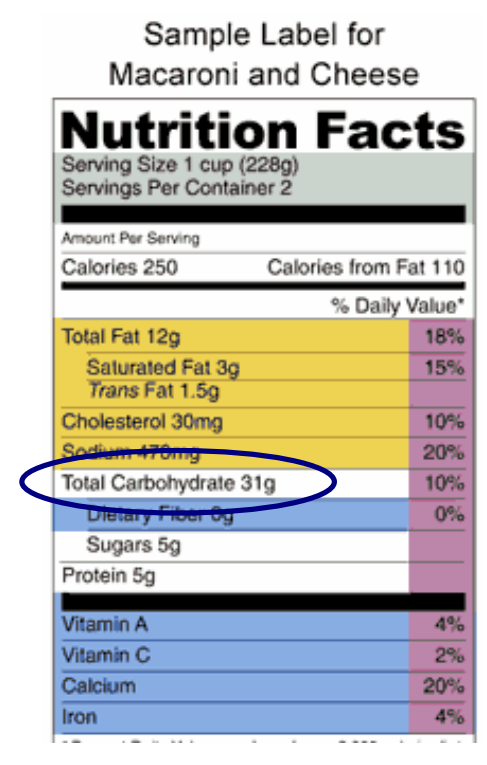




\section{Maintaining a healthy weight}

Maintaining a healthy weight is another way you can help control your blood glucose levels. A plan of healthful eating and staying active to reach and maintain a healthy weight will not only improve your diabetes control, but your general health as well.

Maintaining a healthy weight may help:

- control blood glucose levels by helping your body use insulin better

- reduce high blood pressure

- decrease your risk of heart disease

- improve your general health

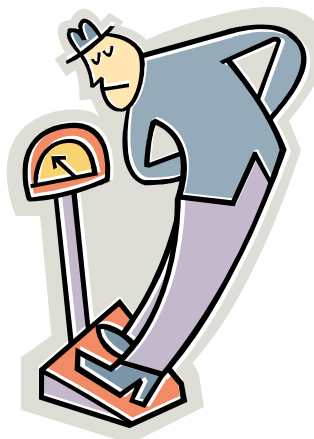

Some people with type 2 diabetes who lose weight may experience near normal or normal blood glucose control. As a result, their doctors may recommend that they stop taking diabetes medicines (pills or insulin). This situation may be temporary or permanent and should be monitored by a doctor.

Maintaining a healthy weight starts with an eating and activity plan that you will enjoy. It should be developed by YOU and your diabetes health care team. This plan should include:

- goals for slow and steady weight loss

- a well-balanced eating plan that you can follow for a lifetime

- an activity program that includes aerobic exercise, like walking, stretching, for flexibility, and strength training, to keep muscles strong

\section{Taking medicine if needed}

Pills and insulin for diabetes are given to keep blood glucose levels within a normal range. Diabetes medicines have various effects. They may:

- $\quad$ stimulate the pancreas to make more insulin

- help decrease insulin resistance

- keep the body from releasing sugar from the liver

- slow down the body's absorption of carbohydrates

Pills: If you have type 2 diabetes, you may need to take one or more pills called oral antidiabetes agents to lower your blood glucose levels. These pills help your body control blood glucose levels in several

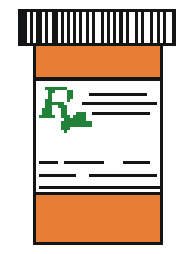
ways. Oral antidiabetes agents help with blood glucose control by:

- giving the pancreas a boost to make the extra insulin your body needs

- helping the insulin you already make work better

- controlling how much glucose your liver makes and puts into your blood

- slowing down the body's absorption of carbohydrates

Insulin: If you have type 1 diabetes, you must take insulin daily, because your pancreas either doesn't make insulin at all or it doesn't make enough.

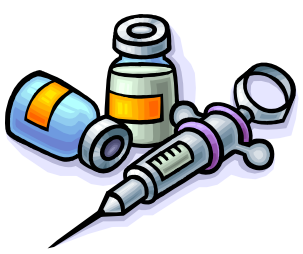

If you have type 2 diabetes, you might need

to take insulin daily if pills alone do not keep your blood glucose in good control.

Sources of Insulin: "Human" insulin is like 
the insulin made in the human body, but it does not come from a human pancreas. It is made by a special chemical process. Animal insulin comes from the pancreas of cows and pigs. These types are available as beef insulin, pork insulin, or a mixture of the two types. Animal insulin has largely been replaced by "human" insulin.

Types of Insulin: There are different types of insulin prescribed to help control blood glucose levels. Since every person with diabetes is different, there is no one type, amount, or combination that is best for everyone. Your doctor will prescribe the type and dose that is right for you.

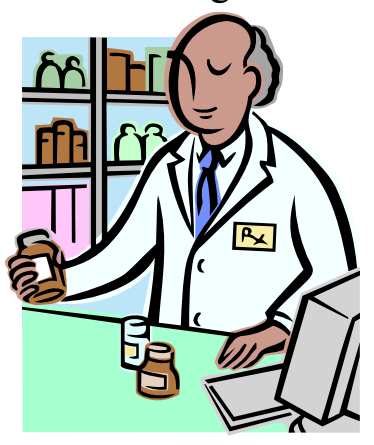

If you take insulin, it's very important that you understand the type you take, how much you take, and how it works in your body (see Table 3 ). Check with your doctor and pharmacist for complete information and instructions about your insulin treatment.

Sometimes it is possible for your blood glucose level to go too low, causing a condition called hypoglycemia (low blood sugar). Hypoglycemia can come on suddenly and must be corrected immediately. If not corrected, low blood glucose may progress to a coma, which is a serious life-threatening condition.

The symptoms of hypoglycemia include:
- shaking

- irritability

- blurred vision

- dizziness
- sweating

- rapid heartbeat

- weakness

- headache
Some people do not experience symptoms until their blood glucose is very low. Be prepared in advance! Ask your doctor what you need to do in case your blood glucose levels go too low.

\section{Recording blood glucose levels}

Keeping track of your blood glucose levels is the best way for you and your doctor to see how well your treatment is working. Based on the results, your doctor can make adjustments in your meal plan, medicine, and/or activity.

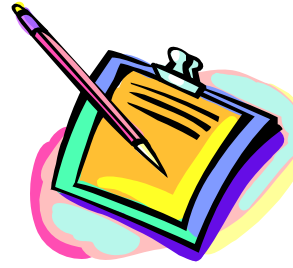

Keeping records of blood glucose is a small job with big payoffs. Remember, keeping blood glucose levels close to the normal range can decrease your chances of having health problems later in life.

\section{Table 3}

\section{Action of Common Types of Insulin}

\begin{tabular}{|l|l|l|l|l|}
\hline Type & $\begin{array}{l}\text { Letter on } \\
\text { Bottle }\end{array}$ & $\begin{array}{l}\text { When it Starts } \\
\text { Working }\end{array}$ & Peak Action & $\begin{array}{l}\text { Total Time it Works } \\
\text { in Your Body }\end{array}$ \\
\hline Rapid Acting & NONE & Less than 15 min & 1 to 2 hours & 3 to 4 hours \\
\hline Short Acting (Regular) & $\mathrm{R}$ & 30 min to 1 hour & 2 to 3 hours & 3 to 6 hours \\
\hline Intermediate Acting (NPH) & $\mathrm{N}$ & 2 to 4 hours & 4 to 10 hours & 10 to 16 hours \\
\hline Long Acting (Ultralente) & $\mathrm{U}$ & 2 to 4 hours & No Peak & 20 to 24 hours \\
\hline * Human insulin works faster than animal insulin. The time ranges given are for human insulin.
\end{tabular}


Your doctor will tell you how often to test your blood glucose and what kind of records to keep. For persons taking multiple insulin injections, the American Diabetes Association (ADA)

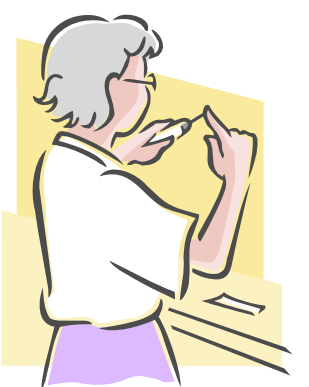
recommends testing blood glucose three or more times a day. For others, they suggest testing often enough to meet blood glucose goals.

Testing in the morning provides fasting blood glucose levels, but does not

let patients know how various foods affect their blood glucose. To get this information, testing also should be done two hours after the first bite of a meal or snack. This should be done at least once a day, or as recommended.

A certified diabetes educator or nurse will show you exactly how to use the blood glucose meter. It's important to keep a logbook of the date, time, and result of each test. Take your logbook with you to each check-up. See Table 4 for a sample logbook entry.

\section{Table 4.}

\section{Sample Log Book}

Mary Jones

\begin{tabular}{|c|c|c|c|c|}
\hline Date & $\begin{array}{c}\text { Before } \\
\text { Breakfast }\end{array}$ & $\begin{array}{c}\text { After } \\
\text { Lunch }\end{array}$ & $\begin{array}{c}\text { After } \\
\text { Dinner }\end{array}$ & Bedtime \\
\hline Jan. 14 & 91 & & 148 & \\
Jan. 15 & 112 & & 162 & 138 \\
Jan. 16 & 100 & & 157 & \\
\hline
\end{tabular}

\section{Staying active}

Staying active is another way you can help yourself. Exercise has many benefits, including:

- helping to control your blood glucose

- lowering the amount of pills or insulin needed (for some people)
- reducing your risk for heart disease

- helping to control blood pressure

- decreasing stress

- improving your general health

Before you begin a regular exercise program as part of your diabetes care, talk with your doctor. Your doctor will recommend a safe and effective program based on your present health and blood glucose control.

The ADA recommends at least 150 minutes per week of moderate-intensity aerobic physical activity and/or at least 90 minutes per week of

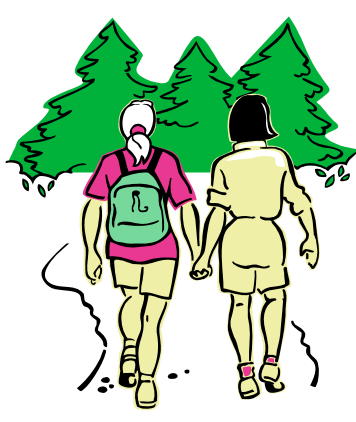

vigorous aerobic exercise. They suggest that the activity be done over at least three days during the week, and that there should be no more than two days in a row without physical activity.

With a doctor's approval, the ADA also encourages resistance exercise to strengthen muscles. Resistance exercise includes lifting free weights and using weight machines. Like aerobic exercise, resistance training helps improve blood glucose control.

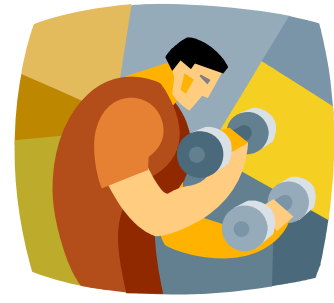

Although everybody is different, there are some general guidelines that all persons with diabetes should follow:

- Wear proper shoes.

- Check your feet daily and after exercise.

- Don't exercise outdoors when it's too hot or too cold.

- Don't exercise if your blood glucose is above a certain number. (Ask your doctor what your blood glucose levels should be to 
exercise safely.)

- Ask your doctor if you should eat a snack before exercising, based on your blood glucose level. This may be the case for persons taking insulin or any oral medication that increases insulin production.

- Wear an identification bracelet to indicate that you have diabetes.

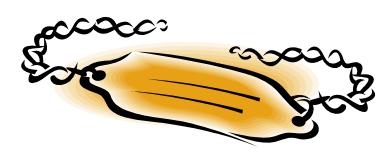

\section{Staying well informed and motivated}

If you have diabetes, the best way to live well and prevent health problems is to stay wellinformed about the disease and motivated enough to practice healthy living.

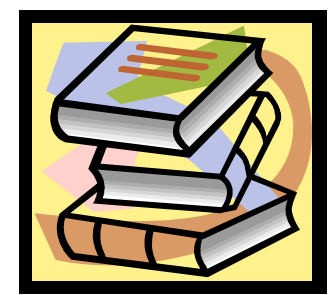

Seek new information regularly, since diabetes treatment and management guidelines change as new research finds better ways to control blood glucose.

As a person with diabetes, it is very important for you to manage your daily activities of healthful eating, taking medicine (if necessary), and exercising. This will help you achieve blood glucose levels as close to normal as is safe and appropriate for you.

You can better care for yourself and practice healthy living with the help and support of family and friends. Some ways they can help you include:

- going to doctor visits with you

- attending special diabetes education classes with you

- eating the same wellbalanced meals you eat

- exercising with you

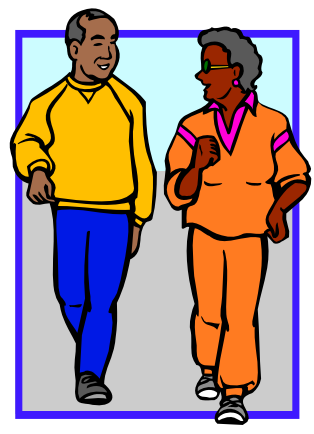

- knowing what to do in case of a diabetic emergency

\section{Possible health problems}

Many people with diabetes live long healthy lives. However, diabetes can cause serious health problems. You can delay or prevent these problems by keeping your blood glucose in good control. Keep in mind that it's easier for some people than others to control blood glucose levels.

High blood glucose can damage small and large blood vessels. Over a long period of time, excess glucose can cause the walls of the blood vessels to thicken. After a while, they can't receive the necessary oxygen and nutrients needed to work properly.

Damage to small blood vessels affects your eyes, kidneys, and nerves. Damage to large blood vessels affects your heart, brain, arms, and legs.

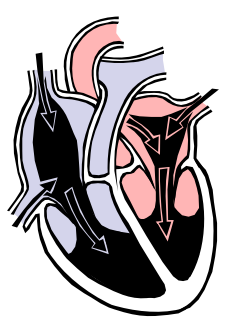

A person with diabetes has a greater chance of having:

- eye disease, called retinopathy

- nerve disease, called neuropathy

- kidney disease, called nephropathy

- heart and blood vessel disease.

- amputations

\section{Reducing the risk of health problems}

Reducing your risk of health problems means taking care of yourself.

- Control your blood glucose levels by eating a healthful diet, taking medicine (pills and/or insulin, if necessary), staying active, keeping daily records of your blood glucose levels, and following any other advice 
suggested by your doctor.

- Be an active member of your Diabetes Care Team. This team is made up of YOU, a doctor, a registered dietitian, a nurse, a pharmacist, and a certified diabetes educator.

\section{Summary}

Although there is no cure for diabetes, it is a disease that can be successfully managed. You can help prevent health problems by playing an active role in your own wellness.

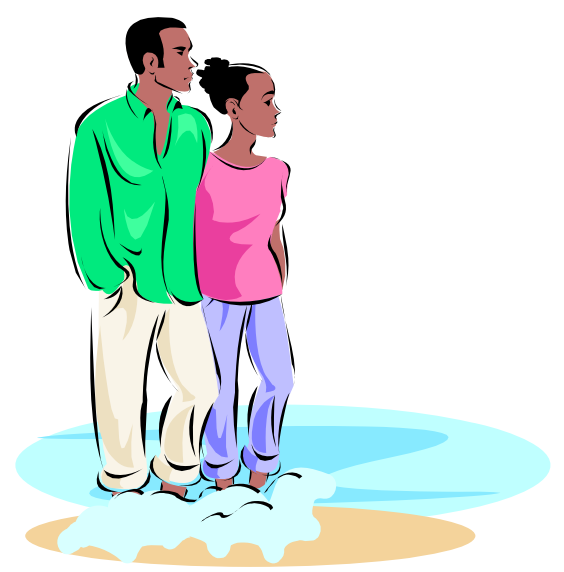

Ask for help when you need it from family, friends, and your health care team.

Take control of your blood glucose and your health by staying informed and doing all you can to take good care of yourself.

\section{For more information}

American Diabetes Association

1701 North Beauregard Street

Alexandria, VA 22311

1-800-342-2383

www.diabetes.org

American Association of Diabetes Educators

$100 \mathrm{~W}$. Monroe, Suite 400

Chicago, IL 60603
$1-800-338-3633$

www.diabeteseducator.org/

The American Dietetic Association

120 South Riverside Plaza

Suite 2000

Chicago, IL 60606-6995

1-800-877-1600

www.eatright.org

Medic Alert Foundation International

2323 Colorado Avenue

Turlock, CA 95382

1-800-633-4298

www.medicalert.org

National Diabetes Education Program

www.ndep.nih.gov

National Institute of Diabetes, Digestive and

Kidney Diseases

www.niddk.nih.gov

Local Resources (check your telephone book for numbers):

Cooperative Extension Service

Health Department

Local Hospitals

\section{Reference}

American Diabetes Association. Standards of Medical Care in Diabetes - 2006 (Position Statement). Diabetes Care 2006;29:S4-S42.

Retrieved July 10, 2006, from

http://care.diabetesjournals.org 